\title{
Identification of an 11T allele in the polypyrimidine tract of intron 8 of the CFTR gene
}

Daniel Kobler, PhD, Hemanshu Modi, BSc, and Barbara Goldman, PhD2006

\begin{abstract}
Purpose: Most of the kits or reagents available for testing for mutations in the cystic fibrosis transmembrane conductance regulator gene include testing for the 5/7/9T polypyrimidine tract, but these methods only screen for three variants in this region: $5 \mathrm{~T}, 7 \mathrm{~T}$, and $9 \mathrm{~T}$. Although such commercial products may not have been designed to screen for rare alleles of the polypyrimidine tract, we demonstrate that at least one of them (Tag-lt ${ }^{\mathrm{TM}}$ Cystic Fibrosis Kit, Tm Bioscience, Toronto, Ontario, Canada) has enough sensitivity to differentiate samples with rare alleles by describing how this product allowed us to detect a previously uncharacterized 11T allele. Methods: A total of 139 banked and anonymized clinical samples from carrier adults and children with cystic fibrosis (The Hospital for Sick Children, Toronto, Canada) were tested and analyzed using the Tag-lt'M Cystic Fibrosis Kit. Results: Two samples displayed allelic ratios for the polypyrimidine tract that were significantly different from the other samples and did not correspond to values expected to be seen for samples with 5T, 7T, or 9T alleles. Further tests with sequencing and an extended Tag-It $\mathrm{t}^{\mathrm{TM}}$ assay confirmed the presence of an $11 \mathrm{~T}$ allele. Conclusion: Although commercial products used in cystic fibrosis testing may not have been designed to screen for rare alleles of the polypyrimidine tract, we demonstrated that at least the Tag-It ${ }^{\mathrm{TM}}$ assay may have enough sensitivity to differentiate samples with such rare alleles, which can then be further analyzed for clarification. Genet Med 2006:8(2):125-128
\end{abstract}

Key Words: CFTR gene, cystic fibrosis, intron 8, polypyrimidine tract

Cystic fibrosis (CF) is one of the most common autosomal recessive disorders among whites, with a prevalence of approximately 1 in 3200 live births. ${ }^{1,2}$ This incidence varies depending on the ethnic group being screened. ${ }^{3,4} \mathrm{CF}$ is caused by mutations in the $230-\mathrm{kb}$ cystic fibrosis transmembrane conductance regulator (CFTR) gene located on the long arm of chromosome 7.5,6 Although more than 1300 CFTR mutations have been reported to date, ${ }^{7}$ most of them are rare. In fact, only approximately 25 of these mutations have an allelic frequency of at least $0.1 \%$ in the general U.S. population. ${ }^{8}$

The demand for CF carrier screening has increased dramatically in response to recommendations made in 2001 by the American College of Medical Genetics, the American College of Obstetricians and Gynecologists, and the National Institutes of Health. ${ }^{8}$ These guidelines outline a standard screening panel covering 25 pan-ethnic CF-causing mutations with an allelic frequency greater than or equal to $0.1 \%$ in the general U.S. population, as well as four CFTR variants that are included as reflex tests. The guidelines have recently been revised ${ }^{9}$ to reduce the recommended panel to 23 mutations.

From the Tm Bioscience Corporation, Toronto, Ontario, Canada.

Daniel Kobler, PhD, Tm Bioscience, 439 University Avenue, Toronto ON, M5G 1Y8, Canada.

Submitted for publication July 29, 2005

Accepted for publication October 31, 2005.

Disclosure statement: All three authors are employees of Tm Bioscience, the manufacturer of the Tag-It ${ }^{\mathrm{TM}}$ Cystic Fibrosis Kit used in this study.

DOI: 10.1097/01.gim.0000200217.85820.47
One of the four variants recommended as reflex test is the 5/7/9T polypyrimidine tract in intron 8 just $5^{\prime}$ of exon 9 . It modulates in cis the phenotypic consequences of the $\mathrm{R} 117 \mathrm{H}$ mutation; $\mathrm{R} 117 \mathrm{H} / 5 \mathrm{~T}$ is associated with $\mathrm{CF}$, and $\mathrm{R} 117 \mathrm{H} / 7 \mathrm{~T}$ is associated with congenital bilateral absence of the vas deferens. ${ }^{10,11}$

To meet the accuracy and throughput demands now required by $\mathrm{CF}$ testing laboratories, diagnostic companies have developed various products, including ELUCIGENE CFPoly-T (Tepnel Diagnostics, Abingdon, Oxon, UK), INNOLiPA CFTR (Innogenetics, Gent, Belgium), and the Tag-It ${ }^{\mathrm{TM}}$ Cystic Fibrosis Kit (Tm Bioscience, Toronto, Ontario, Canada). Most of the available products include testing for the 5/7/9T polypyrimidine tract, but these methods only screen for three variants in this region: 5T, 7T, and 9T. Recently, a 3T, 6T, and $10 \mathrm{~T}$ allele have been reported, ${ }^{12,13,14,15}$ showing that other alleles, although rare, exist and may affect the diagnosis of patients.

Although commercially available products only screen for $5 \mathrm{~T}, 7 \mathrm{~T}$, and $9 \mathrm{~T}$, some may still possess enough specificity to allow for the detection of unusual alleles. Indeed, during the analytic validation of the Tag-It ${ }^{\mathrm{TM}}$ Cystic Fibrosis Kit, a Food and Drug Administration-cleared kit that screens for the three main poly $\mathrm{T}$ alleles (as well as another 39 mutations in the CFTR gene), two samples showed an unexpected behavior that may be the result of the presence of a previously uncharacterized $11 \mathrm{~T}$ allele. 


\section{MATERIALS AND METHODS}

Although this test is described in detail elsewhere, ${ }^{16}$ it is useful to briefly present the basic elements of the Tag- $\mathrm{It}^{\mathrm{TM}}$ assay. Starting with genomic DNA (e.g., extracted from whole blood or blood spots), a single polymerase chain reaction generates 16 amplimers that are subjected to allele-specific primer extension (ASPE) with 86 tagged primers. As alleles are extended, they incorporate biotin-deoxycytidine triphosphate for detection. Tagged products are sorted by hybridization to a universal bead array consisting of 86 color-coded microspheres. Reporter signal is detected on a Luminex xMAP instrument (Luminex Corporation, Austin, TX) providing median fluorescence intensities (MFIs) for each allele. This same type of assay, although with a much lower level of multiplexing, has also been used to detect a panel of thrombophilia-associated single-nucleotide polymorphisms. ${ }^{17}$ The data analysis performed for the polypyrimidine tract starts with the MFIs obtained for the 5T, 7T, and 9T alleles. These values are then transformed into net MFIs by subtraction of the MFI values of a polymerase chain reaction negative control sample (a net MFI is set to 0 if the subtraction leads to a negative value) and used to calculate allelic ratios where allelic ratio $=$ net MFI for an allele/sum of the net MFIs for all three alleles.

Allelic ratios represent the fraction of the total net MFI signal attributed to the presence of a particular allele. By definition, an allelic ratio is between 0 and 1 (included), and the sum of the $5 \mathrm{~T}$ allelic ratio (5T-AR), the $7 \mathrm{~T}$ allelic ratio (7T-AR), and the 9T allelic ratio (9T-AR) is equal to 1 . An allele is considered to be present if its allelic ratio is at least 0.30 (if all three alleles have an allelic ratio of at least 0.30 , the data analysis algorithm provides a "No Call" to mark the ambiguous result).

A sample that is homozygous for the 7T allele will have a 7T-AR at approximately 1 , and 5T-AR and 9T-AR at approximately 0 . Similarly, a sample that has both the 7T and the 9T alleles will have 7T-AR and 9T-AR at approximately 0.5 , and 5T-AR at approximately 0 .
Table 1

Allelic ratio ranges and values from a 139-sample study

\begin{tabular}{lclll}
\hline Samples & Genotype & 5T-AR & 7T-AR & 9T-AR \\
\hline 1 sample & $5 \mathrm{~T} / 5 \mathrm{~T}$ & 0.98 & 0.02 & 0.00 \\
4 samples & $5 \mathrm{~T} / 7 \mathrm{~T}$ & $0.54-0.57$ & $0.42-0.45$ & $0.01-0.01$ \\
4 samples & $5 \mathrm{~T} / 9 \mathrm{~T}$ & $0.49-0.53$ & $0.01-0.02$ & $0.45-0.49$ \\
41 samples & 7T/7T & $0.02-0.03$ & $0.92-0.97$ & $0.01-0.06$ \\
61 samples & 7T/9T & $0.01-0.02$ & $0.43-0.50$ & $0.49-0.55$ \\
26 samples & 9T/9T & $0.01-0.02$ & $0.02-0.04$ & $0.95-0.97$ \\
Sample 97 & $7 \mathrm{~T} / 11 \mathrm{~T}$ & 0.03 & 0.85 & 0.12 \\
Sample 101 & $7 \mathrm{~T} / 11 \mathrm{~T}$ & 0.02 & 0.85 & 0.13 \\
\hline
\end{tabular}

$\mathrm{AR}$, allelic ratio.

\section{RESULTS}

In a study of 139 banked and anonymized clinical samples from carrier adults and children with CF that were provided by The Hospital for Sick Children (Molecular Genetics Laboratory, Department of Pediatric Laboratory Medicine, Toronto, Canada), the ranges described in Table 1 were observed. The genotype for all samples was confirmed by bidirectional dideoxy dye-terminator sequencing.

Two samples within the study (samples 97 and 101) exhibited allelic ratio values somewhat similar to the samples with a 7T/7T genotype (observed in 41 samples) or a 7T/9T genotype (observed in 61 samples), but nevertheless significantly outside of the observed ranges, as shown in Table 1.

After the study, we took advantage of the flexibility inherent to the Tag-It ${ }^{\mathrm{TM}}$ assay that allows the easy expansion of the panel of mutations being screened. By incorporating ASPE primers for an $11 \mathrm{~T}$ allele and a $13 \mathrm{~T}$ allele and two additional Luminex ${ }^{\circledR}$ bead populations, the Tag-It ${ }^{\mathrm{TM}}$ assay was easily expanded to allow for screening of these two poly $\mathrm{T}$ alleles. The expanded assay was then used to reanalyze samples representing all six possible combinations of the 5T, 7T, and 9T alleles, as well as samples 97 and 101. The analysis of the results shown in Table 2 indicates a 7T/11T genotype for samples 97 and 101 (al-

Table 2

Allelic ratio ranges and values from a follow-up study with an expanded assay

\begin{tabular}{|c|c|c|c|c|c|c|}
\hline Samples & Genotype & 5T-AR & 7T-AR & 9T-AR & $11 \mathrm{~T}-\mathrm{AR}$ & 13T-AR \\
\hline 1 sample & $5 \mathrm{~T} / 5 \mathrm{~T}$ & 0.95 & 0.03 & 0.01 & 0.00 & 0.01 \\
\hline 1 sample & $5 \mathrm{~T} / 7 \mathrm{~T}$ & 0.53 & 0.45 & 0.01 & 0.00 & 0.00 \\
\hline 1 sample & $5 \mathrm{~T} / 9 \mathrm{~T}$ & 0.43 & 0.02 & 0.54 & 0.01 & 0.00 \\
\hline 2 samples & $7 \mathrm{~T} / 7 \mathrm{~T}$ & $0.02-0.02$ & $0.96-0.96$ & $0.01-0.01$ & $0.00-0.00$ & $0.00-0.00$ \\
\hline 2 samples & $7 \mathrm{~T} / 9 \mathrm{~T}$ & $0.02-0.02$ & $0.41-0.44$ & $0.53-0.56$ & $0.00-0.01$ & $0.00-0.01$ \\
\hline 1 sample & $9 \mathrm{~T} / 9 \mathrm{~T}$ & 0.01 & 0.02 & 0.95 & 0.01 & 0.01 \\
\hline Sample 97 & $7 \mathrm{~T} / 11 \mathrm{~T}$ & 0.02 & 0.55 & 0.06 & 0.34 & 0.02 \\
\hline Sample 101 & $7 \mathrm{~T} / 11 \mathrm{~T}$ & 0.02 & 0.57 & 0.06 & 0.34 & 0.02 \\
\hline
\end{tabular}

$\mathrm{AR}$, allelic ratio. 
(a)

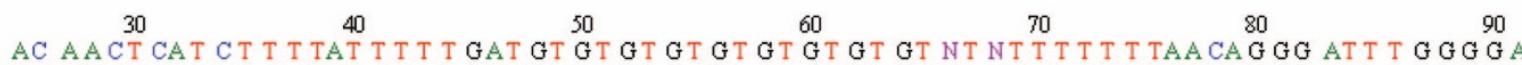

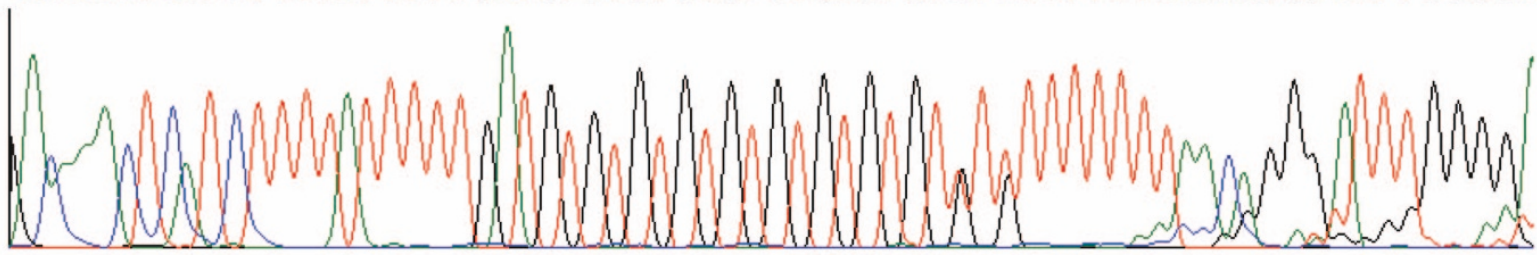

(b)

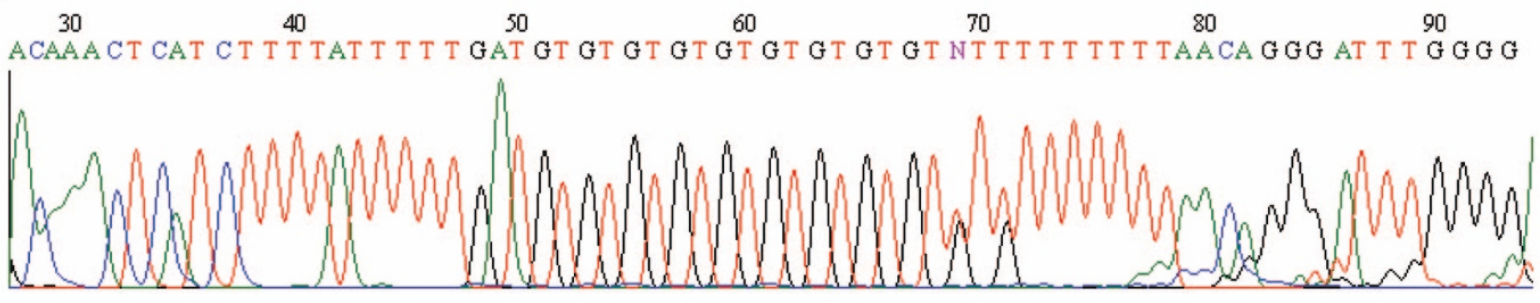

(c)

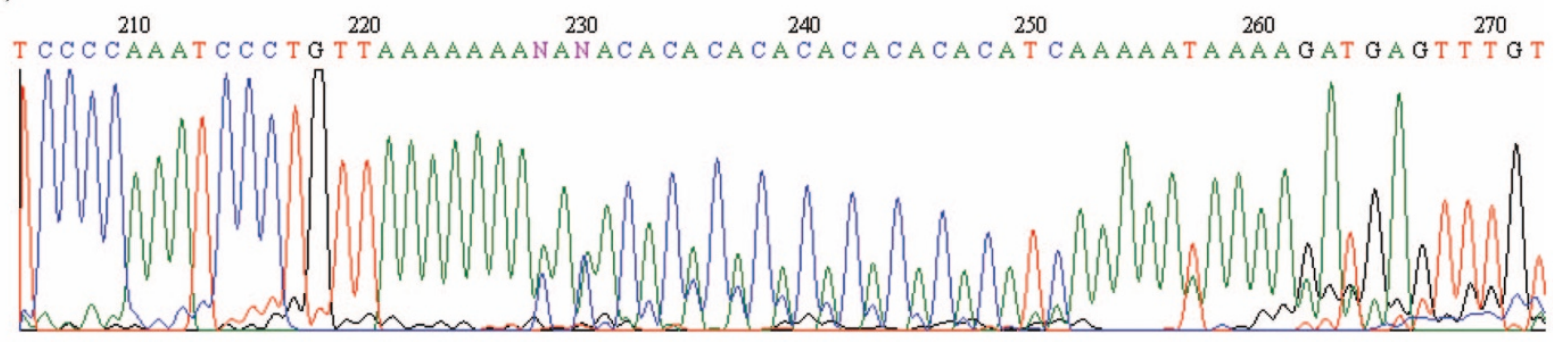

(d)

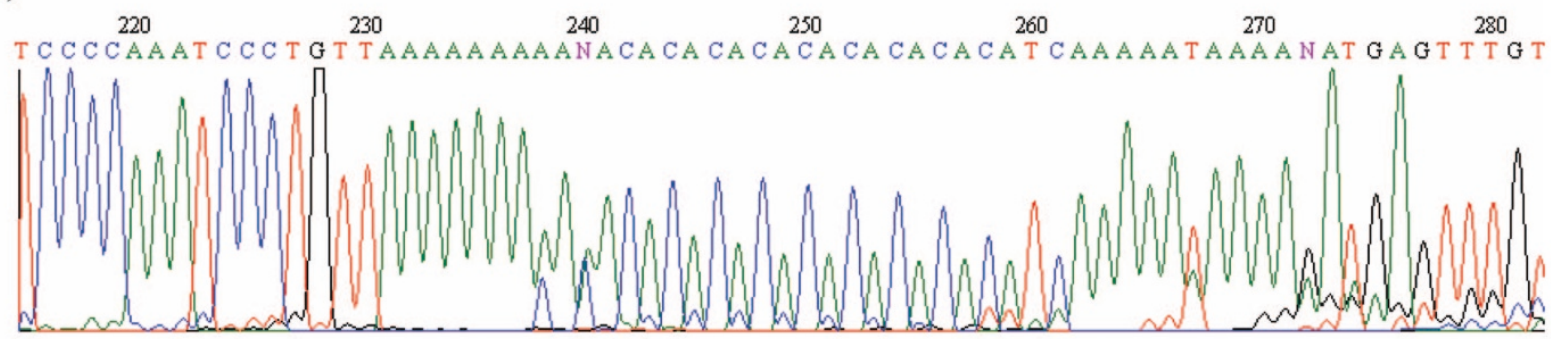

(e)

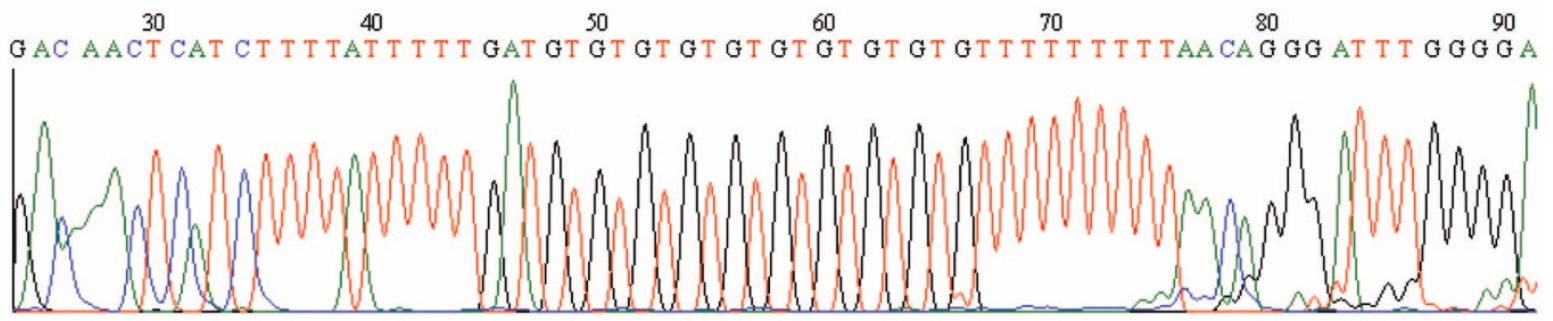

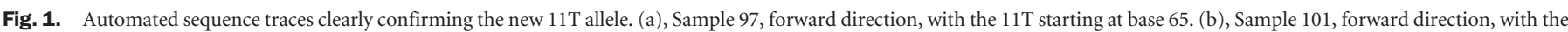

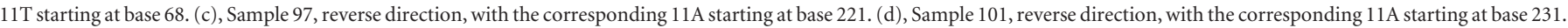

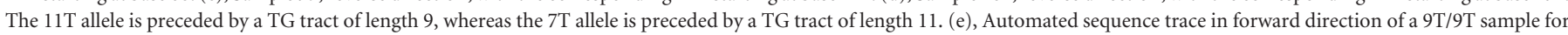
comparison. 
though samples 97 and 101 exhibit higher crossover signal on the 9T allele than the other samples, the 9T-AR remains well below the threshold of 0.30 for the allele to be considered present). Bidirectional sequencing confirmed that both sample 97 and sample 101 have genotype 7T/11T (Fig. 1). Note that for Table 2, the sum of the net MFIs of the five alleles screened in this extended assay is used as the denominator in the calculation of allelic ratios. This explains the significant decrease in the 7T-AR between the two studies when the net MFIs have not significantly changed (data not shown). Note also that the 11T and 13T ASPE primers have not undergone any optimization work, explaining the 11T-AR further away from the ideal value of 0.5 when the $11 \mathrm{~T}$ allele is present.

\section{DISCUSSION}

Although 2 of the 139 samples of the study exhibited this 7T/11T genotype, it was revealed to us after the study that the two samples come from the same individual, who was also homozygous for the $\Delta \mathrm{F} 508$ mutation (the most common of the CF mutations), without any indication of unusual phenotype (for a CF case). Looking at the poly(TG) repeat that is $5^{\prime}$ of the polypyrimidine tract, and that also influences splicing of exon $9,{ }^{18}$ indicates that the sample is 11TG-7T and 9TG-11T. The frequency and phenotypic significance, if any, of this newly discovered $11 \mathrm{~T}$ allele have yet to be established.

Although commercial products used in CF testing may not have been designed to screen for rare alleles of the polypyrimidine tract, we demonstrated that at least the Tag- $\mathrm{It}^{\mathrm{TM}}$ assay may have enough sensitivity to differentiate samples with such rare alleles, which can then be further analyzed for clarification. In addition, the Tag-It ${ }^{\mathrm{TM}}$ platform is open because universally tagged beads are commercially available (FlexMAP ${ }^{\mathrm{TM}}$ beads, Luminex Corporation), making it possible for any laboratory to develop their own assay to complement the Tag- $\mathrm{It}^{\mathrm{TM}}$ products available.

\section{ACKNOWLEDGMENTS}

The authors thank Dr. Brad Popovich for his helpful comments that contributed to this article, and Dr. Peter Ray and The Hospital for Sick Children (Toronto) for providing the samples.

\section{References}

1. Moskowitz SM, Gibson RL, Sternen DL, Cheng E, et al. (Updated 24 August 2004): CFTR-Related Disorders. In GeneReviews at GeneTests: Medical Genetics Information Resource. http://www.genetests.org. Accessed 12 May 2005.

2. Rosenstein BJ, Cutting GR. The diagnosis of cystic fibrosis: a consensus statement. Cystic Fibrosis Foundation Consensus Panel. J Pediatr 1998;132:589-595.

3. Hamosh A, Fitz-Simmons SC, Macek M Jr, Knowles MR, et al. Comparison of the clinical manifestations of cystic fibrosis in black and white patients. J Pediatr 1998; 132:255-259.

4. Kerem B, Chiba-Falek O, Kerem E. Cystic fibrosis in Jews: frequency and mutation distribution. Genet Test 1997;1:35-39.

5. Riordan JR, Rommens JM, Kerem B, Alon N, et al. Identification of the cystic fibrosis gene: cloning and characterization of complementary DNA. Science 1989; 245:1066-1073.

6. Rommens JM, Iannuzzi MC, Kerem B, Drumm ML, et al. Identification of the cystic fibrosis gene: chromosome walking and jumping. Science 1989;245:1059-1065.

7. Cystic Fibrosis Mutation DataBase. http://www.genet.sickkids.on.ca/cftr/. Accessed 12 May 2005.

8. Grody WW, Cutting GR, Klinger KW, Richards CS, et al. Laboratory standards and guidelines for population-based cystic fibrosis carrier screening. Genet Med 2001;3: 149-154.

9. Watson MS, Cutting GR, Desnick RJ, Driscoll DA, et al. Cystic fibrosis population carrier screening: 2004 revision of American College of Medical Genetics mutation panel. Genet Med 2004;6:387-391.

10. Chillon M, Casals T, Mercier B, Bassas L, et al. Mutations in the cystic fibrosis gene in patients with congenital absence of the vas deferens. N Engl J Med 1995;332:14751480 .

11. Kiesewetter S, Macek M, Davis C, Curristin SM, et al. A mutation in CFTR produces different phenotypes depending on chromosomal background. Nat Genet 1993;5: 274-277.

12. Disset A, Michot C, Guittard C, DeGeorges M, et al. Identification of a T3 allele in a CBAVD (congenital bilateral absence of vas deferens) patient: in vitro studies demonstrate tissue specificity of aberrant exon 9 splicing. Belfast: 26th European CF Conference. 2003:4-7.

13. Disset A, Michot C, Harris A, Buratti E, et al. A T3 allele in the CFTR gene exacerbates exon 9 skipping in vas deferens and epididymal cell lines and is associated with Congenital Bilateral Absence of Vas Deferens (CBAVD). Hum Mutat 2005;25:7281.

14. Millson A, Pont-Kingdon G, Page S, Lyon E. Direct molecular haplotyping of the IVS- 8 poly(TG) and polyT repeat tracts in the cystic fibrosis gene by melting curve analysis of hybridization probes. Clin Chem 2005;51:1619-1623.

15. Viel M, Leroy C, DeGeorges M, Claustres M. Novel length variant of the polypyrimidine tract with the splice acceptor site in intron 8 of the CFTR gene: consequences for genetic testing using standard assays. Eur J Hum Genet 2005;13:136-138.

16. Lisle CM, Bortolin S, Kobler D, Fieldhouse D, et al. Cystic fibrosis mutation detection using a bead-based universal array. Clin Chem 2003;49:A15.

17. Bortolin S, Black M, Modi H, Boszko I, et al. Analytical validation of the Tag-It high-throughput microsphere-based universal array genotyping platform: application to the multiplex detection of a panel of thrombophilia-associated single-nucleotide polymorphisms. Clin Chem 2004;50:2028-2036.

18. Cuppens H, Lin W, Jaspers M, Costes B, et al. Polyvariant mutant cystic fibrosis transmembrane conductance regulator genes. The polymorphic (TG)m locus explains the partial penetrance of the $5 \mathrm{~T}$ polymorphism as a disease mutation. J Clin Invest 1998;101:487-496. 